\title{
Three-dimensional fracture analysis of CT specimens with a ductile damage model based on endochronic plasticity theory
}

\author{
C.L. CHOW and X.F. CHEN \\ Department of Mechanical Engineering, University of Michigan-Dearborn, Dearborn, \\ MI 48128-1491, USA
}

Received 11 June 1993; accepted in revised form 17 August 1994

\begin{abstract}
For many fracture problems of practical engineering importance, the three-dimensional effects are significant and a three-dimensional analysis for the problems is thus required. In this paper, an endochronic theory coupled with anisotropic damage is first established, which is actually an elasto-plastic damage theory coupled with isotropic-nonlinear kinematic hardening. The ductile damage evolution equation is derived from the orthogonality rule with a new intrinsic time scale introduced especially for damage evolution. Then, a three-dimensional finite element program incorporating the endochronic damage model is formulated and employed to analyze the widely used $\mathrm{CT}$ fracture specimen. Two failure criteria are proposed for the prediction of crack initiation direction and crack initiation load. From the analysis, significant three-dimensional effects are observed and the crack is estimated to initiate first at the middle of the crack front line. Experiments have been conducted to verify the proposed theory and the results are found to compare well with the theoretical values.
\end{abstract}

\section{Introduction}

For fracture analysis of ductile materials, $J$ is widely employed when plastic deformation at the crack tip cannot be ignored. Actually $J$ 's applicability is based on the existence of the HRR field $[1,2,3]$ when some minimal size requirements are met $[4,5,6]$. However, the characteristic HRR crack tip fields are not always valid. The precise characteristic fields depend on the size of specimen chosen. When the size requirements are not fully satisfied, several two-parameter criteria have been proposed [7-10] as the stress state plays an important role in the fracture process. Consequently, another parameter employed in the two-parameter criteria besides $J$ is a parameter describing the stress state. The $J$-integral approach can only characterize a well-defined macro-crack without taking into account the presence of micro-voids/cracks in a material element. However, any 'real-life' material contains a varying degree and size of micro-voids/cracks. Despite its popularity, ductile characterization using the $J$-integral approach is not considered realistic.

In reality, the crack initiation and subsequent propagation process is a progressive damage accumulation process. Microscopically the microcracks/voids initiate generally at the brittle inclusions, precipitates, the embrittled grain boundaries, or particle-matrix interfaces for ductile failure. Once the microcracks/voids initiate at these sites, they proceed to grow by plastic deformation, and finally these initiated and developed microvoids/cracks coalesce or link with the crack tip or notch root [11]. The stress triaxiality plays a very important factor in microvoid development in addition to the plastic deformation. This is one reason why twoparameter criteria employ the stress triaxiality as the additional parameter with $J$ integral in 
the crack analysis, and different $J_{c}$ are obtained with different specimen geometries because the deformation constraints are different.

As an alternative methodology to the global approach, local approach is introduced to predict crack behavior. In the local approach, the crack initiation and propagation are considered as the failure of material elements in the course of crack propagation ahead of the crack tip and they are treated in a consistent way. In damage mechanics, the material failure is considered as a progressive process depending on the stress and strain histories. When it is applied to analyze the crack problem by local approach, the material ahead of the crack tip experiences large stress and strain histories. The damage will be highly developed at the crack tip due to the stress and strain concentrations, especially in the interior part of the three-dimensional crack front due to high stress triaxiality. In the local approach of a crack problem with damage concept, the damage accumulation is precisely evaluated in the crack tip and then employed for the crack initiation and subsequent propagation analysis. Due to the appearance of these material defects, the material deteriorates and its stiffness reduces especially in the crack tip, thus causing the redistribution of stress near the crack tip. Finally the crack initiates when the local failure criterion is satisfied.

The CT specimen is one of the commonly used specimens in fracture mechanics to evaluate material fracture toughness. One of the requirements for the CT specimen for measuring the fracture toughness is that the thickness must be large enough to ensure the attainment of plain strain deformation mode in most interior parts of the specimen, such that the measured fracture toughness $J_{I C}$ is therefore stable. For a CT specimen whose thickness is not so large and at the same time not thin enough to ensure that the plane stress analysis is applicable, significant three-dimensional effect can be observed from the experiments. The crack initiates first in the middle of the specimen and the crack propagates much faster in the interior part than on the surface, causing the tunneling phenomenon of the crack propagation. Obviously, such a problem cannot be treated as either plane strain or plane stress. These three-dimensional effects prompt the present three-dimensional analysis of the CT specimen and the corresponding crack initiation criterion applicable to three-dimensional analysis.

Due to the complexity of its geometry and the complex deformation state at the near crack tip region relating to elastic-plastic three-dimensional fracture analysis, the information on such three-dimensional analysis of fracture is limited as compared with numerous works on either plane stress analysis or plane strain analysis. Strictly speaking, the real fracture process is three-dimensional in nature. The plane stress analysis or plane strain analysis is only an approximation to the real fracture process when certain conditions are broadly satisfied. Therefore it is necessary to conduct a fully three-dimensional analysis for the detailed investigation of the fracture process at the crack tip when two-dimensional analysis is not applicable.

As to the local approach of fracture coupled with damage, the applications of the approach to two-dimensional analysis are limited [12-14]. And three-dimensional analysis is rarely observed. In this paper, only the crack initiation of a CT specimen with a three-dimensional analysis will be conducted due to the limitation of computer capability. Similar analysis can be readily extended to crack propagation although CPU time needed is much larger due to the plastic unloading etc. 


\section{A ductile damage model based on endochronic plastic theory}

The endochronic theory was first introduced by Valanis $[15,16]$ based on irreversible thermodynamics, where the existence of entropy as a state variable was established for both reversible and irreversible processes by the integrability of the first law of thermodynamics [17]. The essential ingredient in endochronic theory is the intrinsic time scale which is defined as the functional of the plastic history. As the microdefects initiate and develop during the plastic deformation causing deterioration of the material properties and finally the material failure, a damage variable is introduced to describe the effects of these microdefects and to incorporate them in the constitutive equations. Firstly, the effective stress concept and the effective strain concept are introduced in the development of the constitutive equation:

$$
\begin{aligned}
& \tilde{\boldsymbol{\sigma}}=M(\boldsymbol{D}): \boldsymbol{\sigma}, \\
& \tilde{\boldsymbol{\varepsilon}}=M^{-1}(\boldsymbol{D}): \varepsilon,
\end{aligned}
$$

where $\tilde{\sigma}$ and $\tilde{\varepsilon}$ are the effective stress and effective strain, respectively, and $M(D)$ is the fourth order damage effect tensor.

The intrinsic time scale is now defined as the functional of the effective plastic deformation history instead of the plastic deformation history because the damage effects are considered.

$$
\begin{aligned}
\mathrm{d} z & =\frac{\mathrm{d} \zeta}{f(\zeta)}, \\
\mathrm{d} \zeta & =\left(\mathrm{d} \tilde{e}^{p}: \mathrm{d} \tilde{e}^{p}\right)^{1 / 2},
\end{aligned}
$$

where $f(\zeta)$ is the hardening function which is equal or larger than 1 for a hardening material.

As frequently used in the endochronic theory, a linear relation between the rate of an internal variable and its thermodynamic force based on the Onsanger's principle is assumed. Through some mathematical deduction, the incremental stress-strain form of endochronic plastic theory coupled with damage is obtained as [17]

$$
\begin{aligned}
\mathrm{d} \tilde{\boldsymbol{\sigma}} & =2 \mu_{0}\left(\mathrm{~d} \tilde{\boldsymbol{\varepsilon}}-\mathrm{d} \tilde{\boldsymbol{\varepsilon}}^{p}\right)+\lambda_{0}(\boldsymbol{I}: \mathrm{d} \tilde{\boldsymbol{\varepsilon}}) \boldsymbol{I} \\
& =2 \mu_{0} \mathrm{~d} \tilde{\boldsymbol{\varepsilon}}+\lambda_{0}(\boldsymbol{I}: \mathrm{d} \tilde{\boldsymbol{\varepsilon}}) \boldsymbol{I}-\frac{2 \mu_{0}(\tilde{\boldsymbol{S}}-\boldsymbol{r})\langle(\tilde{\boldsymbol{S}}-\boldsymbol{r}): \mathrm{d} \tilde{\boldsymbol{e}}\rangle}{C\left(S_{y}^{0}\right)^{2} f^{2}(\zeta)} \Gamma,
\end{aligned}
$$

where

$$
C=1+\rho_{1}(0)+\frac{(\tilde{\boldsymbol{S}}-\boldsymbol{r}): \boldsymbol{h}^{*}}{S_{y}^{0} f^{2}(\zeta)}+\frac{S_{y}^{0} f^{\prime}}{2 \mu_{0}}
$$

and

$$
h^{*}=\int_{0}^{z} \frac{\mathrm{d} \rho_{1}}{\mathrm{~d} z}\left(z-z^{\prime}\right) \frac{\mathrm{d} \tilde{e}^{p}}{\mathrm{~d} z^{\prime}} \mathrm{d} z^{\prime},
$$


and

$$
\begin{aligned}
\Gamma=1 & \text { when }\|\tilde{\boldsymbol{S}}-\boldsymbol{r}\|=S_{y}^{0} f(\zeta) \\
& \text { and }(\tilde{\boldsymbol{S}}-\boldsymbol{r}): \mathrm{d} \tilde{\varepsilon}>0 \quad \text { for permissible plastic deformation } \\
\Gamma=0 & \text { when }\|\tilde{\boldsymbol{S}}-\boldsymbol{r}\|<S_{y}^{0} f(\zeta) \text { for material elastic region } \\
& \text { or when }\|\tilde{\boldsymbol{S}}-\boldsymbol{r}\|=S_{y}^{0} f(\zeta) \\
& \text { and }(\tilde{\boldsymbol{S}}-\boldsymbol{r}): \mathrm{d} \tilde{\boldsymbol{\varepsilon}} \leqslant 0 \quad \text { for material elastic unloading, }
\end{aligned}
$$

where $r(z)$ is the 'back stress' as in the classical plasticity theory and describes the translation of the center of yielding surface while the hardening function $f(\zeta)$ describes the dilatation of the yielding surface. In the endochronic theory, the evolution of $r(z)$ is nonlinear and can be characterized as nonlinear kinematic hardening.

In the endochronic theory developed before, the effects of damage on the mechanical behavior are incorporated with $M(D)$ through the concept of effective stress and effective strain. The next key question to introduce the damage effects is to choose appropriate forms of $\boldsymbol{M}(\boldsymbol{D})$. In what follows, we shall discuss the choice of damage effective tensor $\boldsymbol{M}(\boldsymbol{D})$.

The damage effective tensor is assumed in the following form

$$
M=\phi^{-1}=(1-\mathcal{D})^{-1}
$$

where $\mathcal{D}$ must be a fourth order tensor with the same symmetric properties as $\boldsymbol{M}$, and may be called the generalized damage variable. Following the conventional choice in damage mechanics for practical reasons, a symmetric tensor $D$ of order two is employed and the relationship between $\mathcal{D}$ and $D$ is chosen as

$$
\mathcal{D}_{i j k l}=\frac{1}{4}\left(\delta_{i k} D_{j l}+\delta_{i l} D_{j k}+\delta_{j k} D_{i l}+\delta_{j l} D_{i k}\right)
$$

from the theory of higher algebra [18].

With Voigt's notation, (1) can be written in matrix form

$$
\{\tilde{\sigma}\}=[M(D)]\{\sigma\}
$$

where $[M]$ is a $6 \times 6$ matrix which is an alternative representative form of the fourth order tensor $M$ due to its symmetric property. For the same reason, $\mathcal{D}$ may be written in a $6 \times 6$ matrix form. In the principal coordinate system of damage variable $D$

$$
[\mathcal{D}]=\left[\begin{array}{ccccc}
D_{1} & & & & \\
& D_{2} & & & \\
& & D_{3} & & \\
& & & \frac{D_{2}+D_{3}}{2} & \\
& & & & \\
& & & & \frac{D_{1}+D_{3}}{2} \\
& & & & \\
& & & & \frac{D_{1}+D_{3}}{2}
\end{array}\right]
$$


From (8) the damage effective tensor $M(D)$ in the principal coordinate system of damage is expressed as

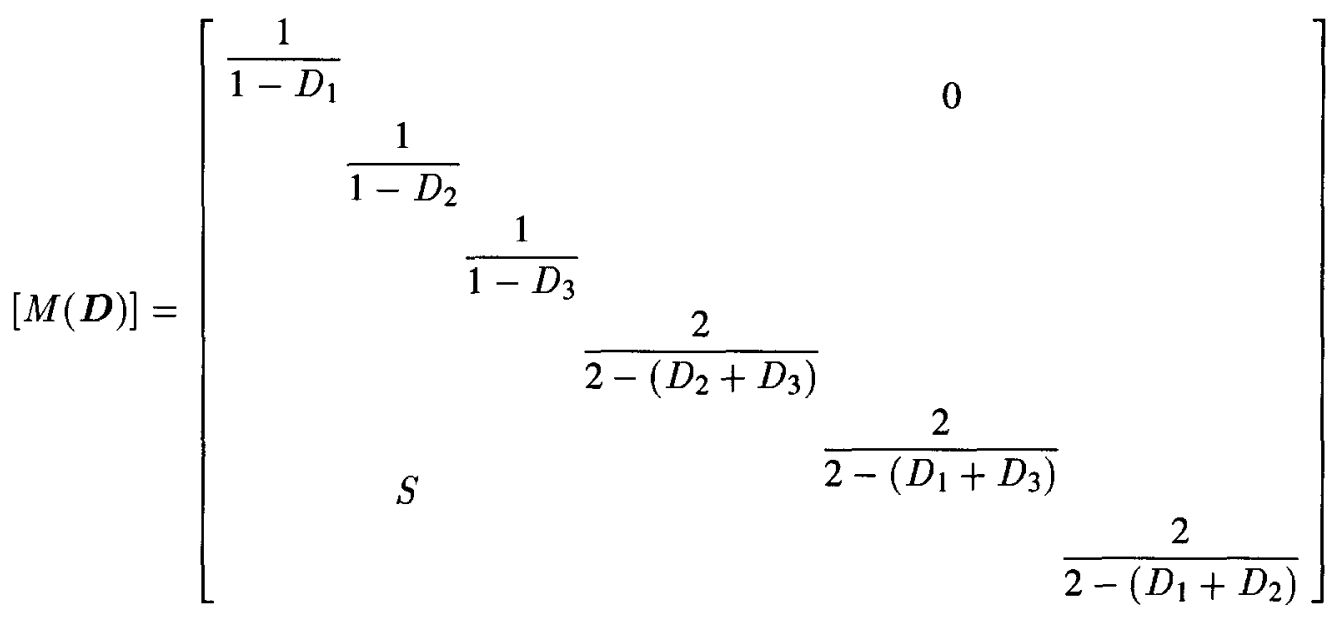

$M(D)$ 's representation in an arbitrary coordinate system could be easily obtained by the tensor transformation.

Ductile damage evolution is proceeded by the plastic deformation. Therefore it is more appropriate to use the intrinsic time scale to describe damage evolution than the commonly used time scale. The intrinsic time scale introduced before is however not directly applicable to the description of damage evolution because the mechanism of damage evolution is different. For ductile damage evolution, the stress state plays a very important role as indicated in $[11,19]$. Therefore a new intrinsic time scale especially defined for damage evolution is introduced as

$$
\mathrm{d} z_{d}=f_{R}(R) \mathrm{d} \zeta / f_{d}(\zeta),
$$

where the index $\mathrm{d}$ of $f_{d}(\zeta)$ represents the effect on damage evolution. The main reason why the intrinsic time measure $\zeta$ is used here is that the evolution of ductile damage is closely related to the plastic deformation and $\zeta$ is a measure in the effective plastic strain space. Because damage evolution is also closely related to the stress state besides plastic deformation, $f_{R}(R)$ is introduced to take this effect into account where $R$ is a parameter to represent stress state, for example the stress triaxiality. The stress triaxiality is defined as $\exp \left(1.5 \tilde{\sigma}_{m} / \tilde{\sigma}_{e q}\right)$ where $\tilde{\sigma}_{m}$ is the effective hydrostatic stress and $\tilde{\sigma}_{e q}$ is the effective equivalent stress.

Damage evolution is a dissipative process based on irreversible thermodynamics. In the following, the relation between the damage evolution and damage dissipation is to be established. If damage dissipation is not coupled with mechanical dissipation, the damage dissipation 'power' to $z_{d}$ must satisfy the following dissipation inequality

$$
\boldsymbol{Y}: \frac{\mathrm{d} \boldsymbol{D}}{\mathrm{d} z_{d}}=\boldsymbol{\Phi}(\boldsymbol{Y}) \geqslant 0,
$$

where $\Phi$ is the damage dissipation 'power'. Then, from the orthogonality rule for dissipative process [20], the damage evolution can be written as

$$
\frac{\mathrm{d} \boldsymbol{D}}{\mathrm{d} z_{d}}=\lambda_{d} \frac{\partial \Phi}{\partial \boldsymbol{Y}},
$$


where

$$
\lambda_{d}= \begin{cases}1 & \text { when } \Phi=\Phi_{\max } \text { and } \frac{\partial \Phi}{\partial \boldsymbol{Y}}: \mathrm{d} \boldsymbol{Y}>0 \\ 0 & \text { when } \Phi<\Phi_{\max } \text { or } \Phi=\Phi_{\max } \text { and } \frac{\partial \Phi}{\partial \boldsymbol{Y}}: \mathrm{d} \boldsymbol{Y} \leqslant 0,\end{cases}
$$

where $\Phi_{\max }$ is the maximum value of $\Phi$.

If we choose the dissipation power $\Phi$ in the following expression

$$
\Phi=\left[\frac{1}{2} \boldsymbol{Y}: \boldsymbol{J}: \boldsymbol{Y}\right]^{1 / 2},
$$

then the damage evolution equation is derived from (15)

$$
\frac{\mathrm{d} \boldsymbol{D}}{\mathrm{d} z_{d}}=\frac{\boldsymbol{J}: \boldsymbol{Y}}{2\left(\frac{1}{2} \boldsymbol{Y}: \boldsymbol{J}: \boldsymbol{Y}\right)^{1 / 2}} H\left(z_{d}-z_{d}^{0}\right)
$$

where

$$
\begin{aligned}
& H(z)=1 \text { for } z>0 \\
& H(z)=0 \text { for } z \leqslant 0 .
\end{aligned}
$$

Here the threshold condition of damage evolution $z_{d}^{0}$ is introduced.

The damage strain energy release rate is the thermodynamic conjugate force to damage variable, and is defined as $[14,21]$

$$
\boldsymbol{Y}=\boldsymbol{\sigma}:\left[\tilde{\boldsymbol{E}}^{-1}: \boldsymbol{M}^{-1}(\boldsymbol{D}): \frac{\partial \boldsymbol{M}(\boldsymbol{D})}{\partial \boldsymbol{D}}\right]^{s}: \sigma .
$$

From (8), we have

$$
\frac{\partial \boldsymbol{M}(\boldsymbol{D})}{\partial \boldsymbol{D}}=\frac{\partial(1-\mathcal{D})^{-1}}{\partial \boldsymbol{D}}=\boldsymbol{M}(\boldsymbol{D}): \frac{\partial \mathcal{D}}{\partial \boldsymbol{D}}: \boldsymbol{M}(\boldsymbol{D}) .
$$

With (12), the $\boldsymbol{Y}$ can be calculated from (19). As Voigt's notation for $\boldsymbol{Y}$ is employed, $\boldsymbol{Y}$ is represented by a vector $\{Y\}$. In the principal coordinate system of damage, the components of $\{Y\}$ are

$$
\begin{aligned}
& Y_{1}=\frac{1}{E}\left(\frac{\sigma_{1}^{2}}{V_{11}^{3}}-\frac{\nu \sigma_{1} \sigma_{2}}{V_{11}^{2} V_{22}}-\frac{\nu \sigma_{1} \sigma_{3}}{V_{11}^{2} V_{33}}+\frac{(1+\nu) \sigma_{5}^{2}}{V_{31}^{3}}+\frac{(1+\nu) \sigma_{6}^{2}}{V_{12}^{3}}\right), \\
& Y_{2}=\frac{1}{E}\left(-\frac{\nu \sigma_{2} \sigma_{1}}{V_{22}^{2} V_{11}}+\frac{\sigma_{2}^{2}}{V_{22}^{3}}-\frac{\nu \sigma_{2} \sigma_{3}}{V_{22}^{2} V_{33}}+\frac{(1+\nu) \sigma_{4}^{2}}{V_{23}^{3}}+\frac{(1+\nu) \sigma_{6}^{2}}{V_{12}^{3}}\right), \\
& Y_{3}=\frac{1}{E}\left(-\frac{\nu \sigma_{1} \sigma_{3}}{V_{33}^{2} V_{11}}-\frac{\nu \sigma_{3} \sigma_{2}}{V_{33}^{2} V_{22}}+\frac{\sigma_{3}^{2}}{V_{33}^{3}}+\frac{(1+\nu) \sigma_{4}^{2}}{V_{23}^{3}}+\frac{(1+\nu) \sigma_{5}^{2}}{V_{31}^{2}}\right),
\end{aligned}
$$



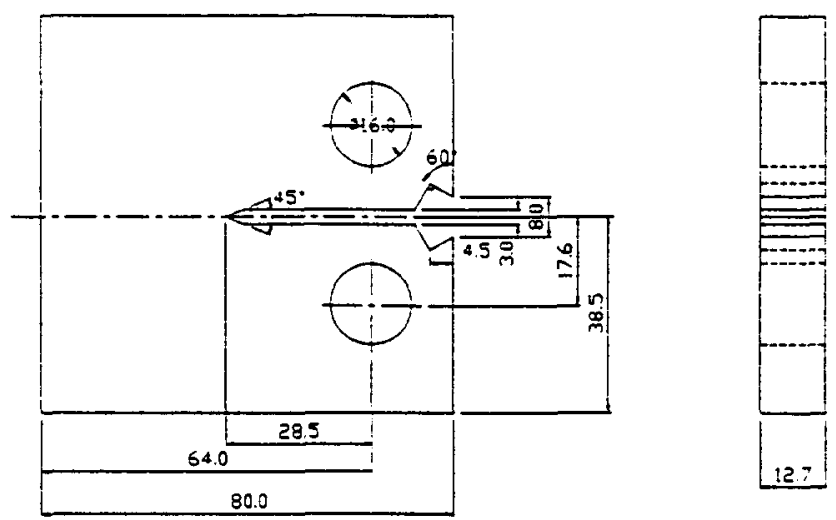

Fig. 1. Geometry of CT specimen $(12.7 \mathrm{~mm})$ material: Al Alloy $2024-\mathrm{T} 351$.

$$
\begin{aligned}
Y_{4}= & \frac{1}{2 E}\left[\left(-\frac{\nu}{V_{11} V_{22} V_{23}}-\frac{\nu}{V_{11} V_{33} V_{23}}\right) \sigma_{1} \sigma_{4}+\left(\frac{1}{V_{22}^{2} V_{23}}-\frac{\nu}{V_{22} V_{33} V_{23}}\right) \sigma_{2} \sigma_{4}\right. \\
& +\left(\frac{1}{V_{33}^{2} V_{23}}-\frac{\nu}{V_{22} V_{33} V_{23}}\right) \sigma_{3} \sigma_{4}+\frac{1+\nu}{V_{23}^{2} V_{22}} \sigma_{2} \sigma_{4}+\frac{1+\nu}{V_{23}^{2} V_{33}} \sigma_{3} \sigma_{4} \\
& \left.+\frac{1+\nu}{V_{31}^{2} V_{12}} \sigma_{5} \sigma_{6}+\frac{1+\nu}{V_{12}^{2} V_{31}} \sigma_{5} \sigma_{6}\right]
\end{aligned}
$$$$
Y_{5}=\frac{1}{2 E}\left[\left(\frac{1}{V_{11}^{2} V_{31}}-\frac{\nu}{V_{11} V_{33} V_{31}}\right) \sigma_{1} \sigma_{5}+\left(-\frac{\nu}{V_{11} V_{22} V_{31}}-\frac{\nu}{V_{22} V_{33} V_{31}}\right) \sigma_{2} \sigma_{5}\right.
$$$$
+\left(\frac{1}{V_{33}^{2} V_{31}}-\frac{\nu}{V_{11} V_{33} V_{31}}\right) \sigma_{3} \sigma_{5}+\frac{1+\nu}{V_{23}^{2} V_{12}} \sigma_{6} \sigma_{4}+\frac{1+\nu}{V_{12}^{2} V_{23}} \sigma_{6} \sigma_{4}
$$$$
\left.+\frac{1+\nu}{V_{31}^{2} V_{11}} \sigma_{5} \sigma_{1}+\frac{1+\nu}{V_{31}^{2} V_{33}} \sigma_{5} \sigma_{3}\right]
$$$$
Y_{6}=\frac{1}{2 E}\left[\left(\frac{\nu}{V_{11}^{2} V_{12}}-\frac{\nu}{V_{11} V_{22} V_{12}}\right) \sigma_{1} \sigma_{6}+\left(\frac{1}{V_{22}^{2} V_{12}}-\frac{\nu}{V_{22} V_{11} V_{12}}\right) \sigma_{2} \sigma_{6}\right.
$$

$$
\begin{aligned}
& +\left(\frac{\nu}{V_{11} V_{33} V_{23}}-\frac{\nu}{V_{22} V_{33} V_{12}}\right) \sigma_{3} \sigma_{6}+\frac{1+\nu}{V_{23}^{2} V_{31}} \sigma_{5} \sigma_{4}+\frac{1+\nu}{V_{31}^{2} V_{23}} \sigma_{5} \sigma_{4} \\
& \left.+\frac{1+\nu}{V_{12}^{2} V_{11}} \sigma_{1} \sigma_{6}+\frac{1+\nu}{V_{12}^{2} V_{22}} \sigma_{1} \sigma_{6}\right]
\end{aligned}
$$

where $V_{11}=1-D_{1} ; V_{22}=1-D_{2} ; V_{33}=1-D_{3} ; V_{23}=1-\left(D_{2}+D_{3}\right) / 2 ; V_{13}=$ $1-\left(D_{1}+D_{3}\right) / 2$ and $V_{12}=1-\left(D_{1}+D_{2}\right) / 2 . D_{1}, D_{2}$ and $D_{3}$ are three principal values of damage $D$. 

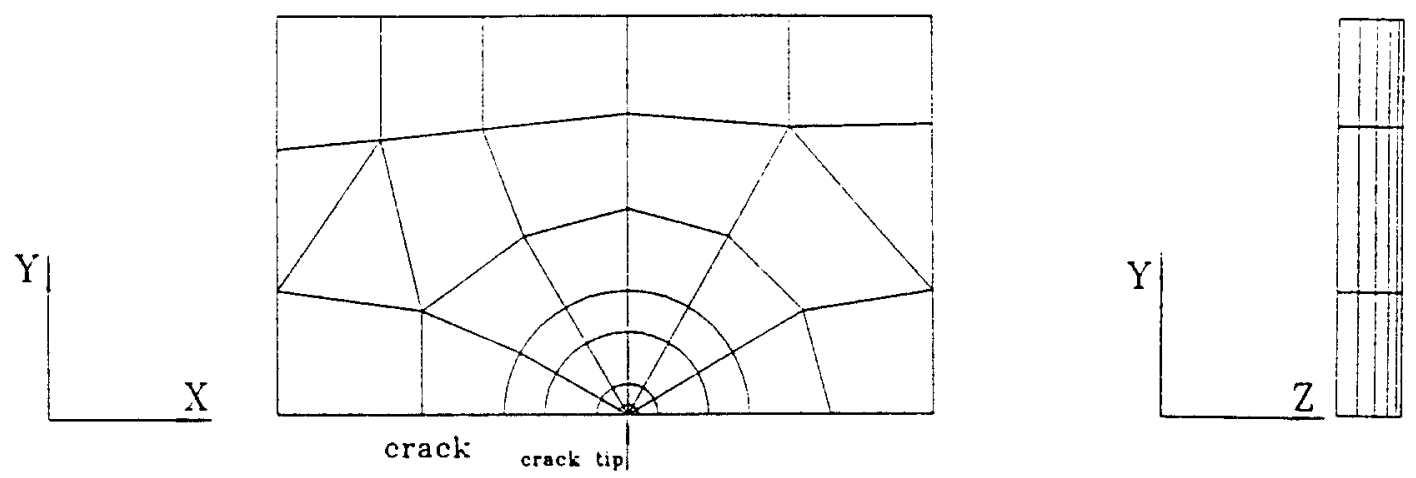

Fig. 2. Finite element mesh of CT specimen.

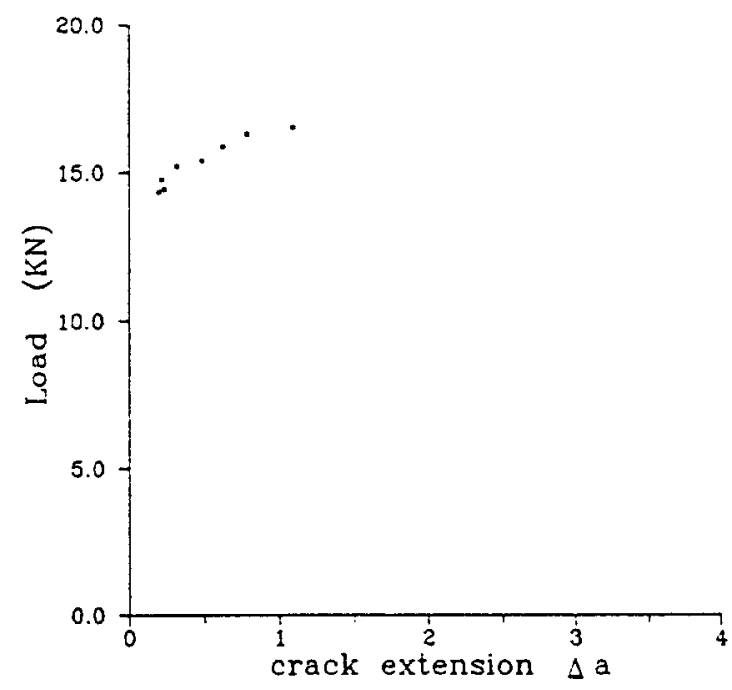

Fig. 3. Load-crack extension $\Delta a$ diagram.

In an arbitrary coordinate system, the components of $Y$ can be readily evaluated from the tensor transformation law. It is of interest to note that the components $Y_{4}, Y_{5}$ and $Y_{6}$ of $Y$ are non-zero in the principal coordinate system of damage if it is not coincident with that of the stress tensor. This is important in the case of nonproportional loading where the principal directions of stress change during the loading process and are not coincident with those of damage. According to the damage evolution equation, the principal directions of the damage increment during nonproportional loading will not be coincident with those of damage, so that the subsequent principal directions of damage will rotate correspondingly.

\section{The finite element formulation}

With the development of the endochronic plastic constitutive equations coupled with damage and the damage evolution equations, the next step is to employ this damage model to characterize fracture in engineering structures under service loading. Since the finite element method has been shown to be a versatile numerical tool in engineering analysis, the method is therefore chosen to analyze the distributions of stress, strain and damage, and to predict the failure behavior of the structures. 
The procedures in formulating the finite element analysis when damage is considered are similar in principle to the conventional FEM analysis. Since stiffness matrix $[K]$ is expressed as a function of transformation $[B]$ and the stress-strain matrix $[C]$ :

$$
[K]=\int_{v}[B]^{T}[C][B] \mathrm{d} V,
$$

where $[C]$ is associated with the material properties while $[B]$, with the element chosen for analysis. Only $[C]$ therefore needs to be modified in order to include the damage effects in stiffness matrix. The effective elastic relation may be written in matrix form

$$
\{\mathrm{d} \tilde{\sigma}\}=\left[C^{e}\right]\left\{\mathrm{d} \tilde{\varepsilon}^{e}\right\} .
$$

By incorporating the endochronic plastic constitutive equation (5) and the damage evolution equation, the above equation becomes

$$
\{\mathrm{d} \tilde{\sigma}\}=\left[C^{*}\right]\{\mathrm{d} \tilde{\varepsilon}\},
$$

where

$$
\left[C^{*}\right]=\left[C^{e}\right]-2 \mu_{0} \frac{\{\hat{\boldsymbol{S}}\}\{\hat{\boldsymbol{S}}\}}{C\left(S_{y}^{0}\right)^{2} f^{2}(\zeta)}
$$

and $\hat{S}$ denotes $(\tilde{\boldsymbol{S}}-\boldsymbol{r})$. Since $[C]$ is the conventional real stress-strain matrix and $\left[C^{*}\right]$ in (29) is the effective stress-strain matrix, it is desirable to transform (29) and express it in terms of the conventional real stress-strain matrix for ease of implementation with the finite element analysis. Equation (29) may thus be deduced as

$$
\{\mathrm{d} \sigma\}=\left[\tilde{C}^{*}\right]\{\mathrm{d} \varepsilon\},
$$

where

$$
\begin{aligned}
& {\left[\tilde{C}^{*}\right]=[M]^{-1}([I]+[Q])\left([I]-\left[C^{*}\right][P]\right)^{-1}\left[C^{*}\right][M]^{T,-1},} \\
& {[Q]=\frac{B_{0}}{f_{d}(\zeta)} f_{R}(R)[M] \frac{\partial[M]^{-1}}{\partial\{D\}^{T}} \frac{\partial \Phi}{\partial\{Y\}^{T}}[M]\{\sigma\}\{\hat{\boldsymbol{S}}\}} \\
& {[P]=\frac{B_{0}}{f_{d}(\zeta)} f_{R}(R) \frac{\partial[M]^{T,-1}}{\partial\{D\}^{T}} \frac{\partial \Phi}{\partial\{Y\}^{T}}\{\varepsilon\}\{\hat{\boldsymbol{S}}\}}
\end{aligned}
$$

and

$$
B_{0}=\frac{1}{2 \mu_{0}(C-1) S_{y}^{0} f(\zeta)}
$$

Because of the symmetric property of the specimen and applied load as shown in Fig. 1, only one-quarter of the specimen needs to be analyzed. The three-dimensional finite element mesh is created in such a way that a two-dimensional finite element mesh is first created, this mesh is then used to generate three-dimensional finite element mesh by duplicating it in the 


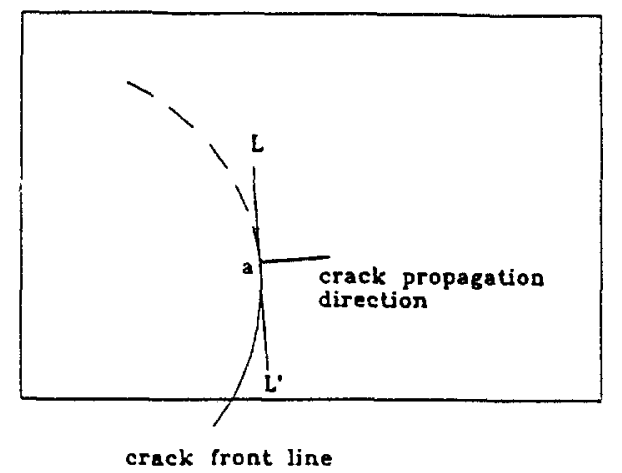

Fig. 4. Schematic definition of the plane perpendicular to the crack front line.

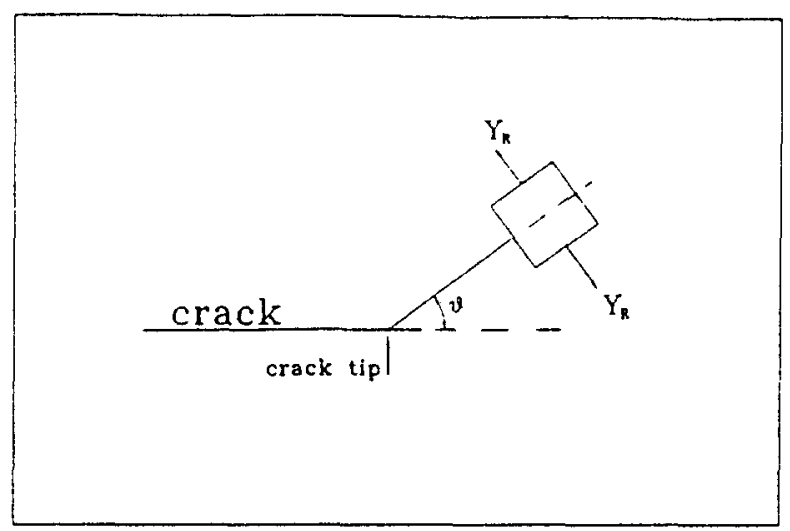

Fig. 5. Definition of $Y_{R}$ at the crack tip.

thickness direction of the specimen as shown in Fig. 2. There are five finite element layers across the half thickness of the specimen. The size of the element in the thickness direction is larger in the interior part of the thickness and decreases progressively to the surface as the variation of stress, strain and other physical quantities in the thickness direction is larger in the surface than in the interior part. In the analysis, a total of 220 8-20 node brick finite elements was employed, and radial elements were used in the crack tip in order to easily obtain the angular and radial distribution of the field quantities of interest in a plane parallel to the surface. The boundary conditions on the symmetric planes are treated by restraining all the nodes in the middle plane ( $x-y$ plane) in $z$-direction displacement, and all the nodes on $x-z$ plane in $y$-direction displacement except the crack region.

Because the material behavior is nonlinear, the step-by-step incremental analysis scheme is employed. The first loading step is comparatively larger till the appearance of the yielding at the crack tip. The loading step is then reduced and applied incrementally until the failure criterion is reached at the crack tip for crack initiation. As the coupling effects of damage and plastic deformation have been taken into account in the constitutive equations used in the analysis, the damage will initiate and develop in the specimen during the loading process. The appearance of damage due to material deterioration will thus cause the decrease of material stiffness, and redistribute the stress field. These effects are especially serious near the crack tip where the damage is localized and highly developed. 


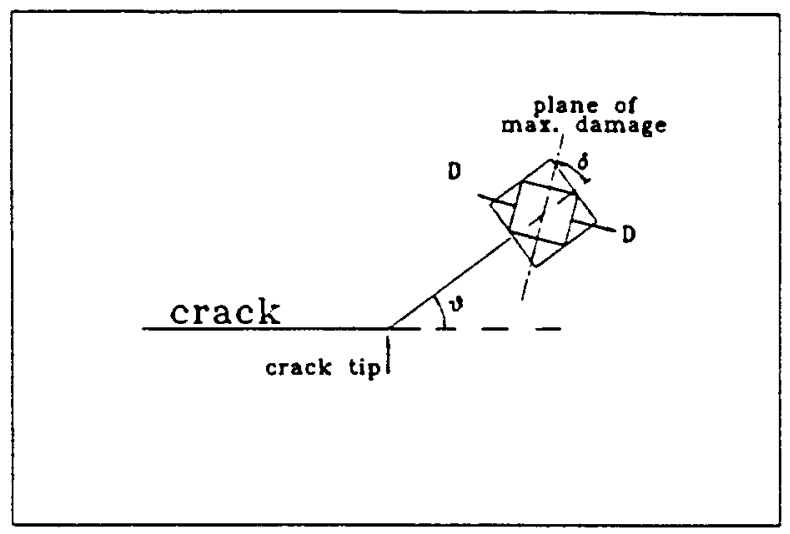

Fig. 6. Definition of $\delta$ at the crack tip.
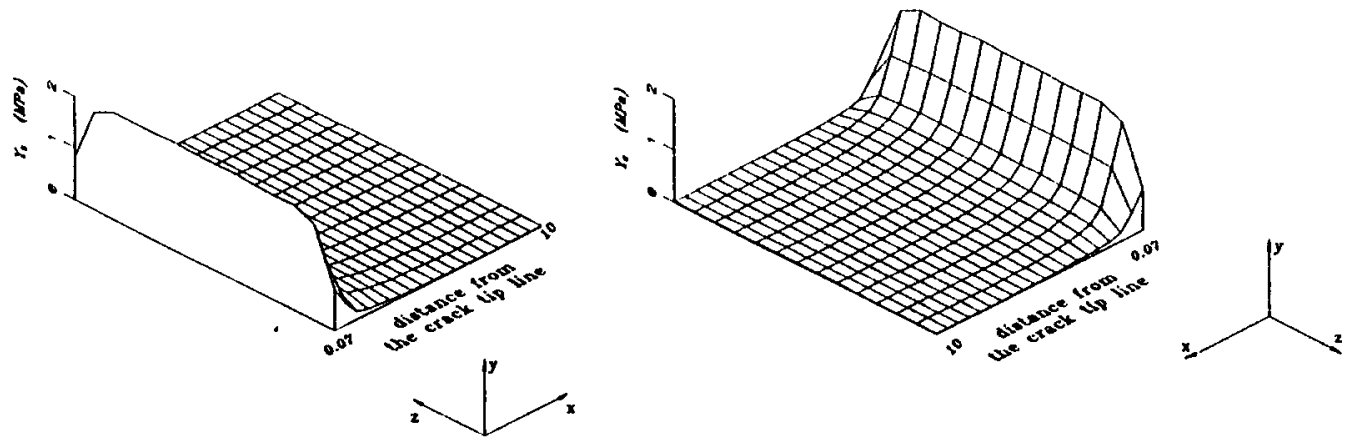

Fig. 7. Distribution of $Y_{R}$ in the crack extension plane CT specimen at the load of $7.63 \mathrm{kN}$.

\section{Experimental investigation}

The geometry of CT specimens chosen is in accordance with the ASTM standard E399-83. The thickness of the material is $12.7 \mathrm{~mm}$. The geometry of the specimen is shown in Fig. 1. Eight specimens have been manufactured for the study. After the CT specimens have been prepared,
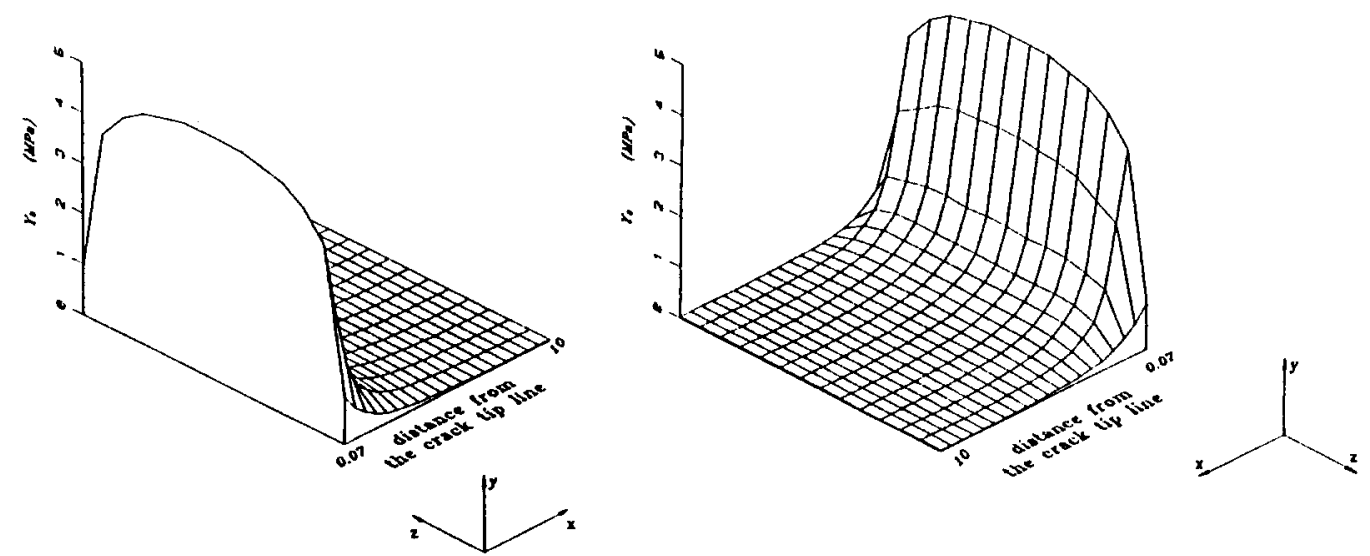

Fig. 8. Distribution of $Y_{R}$ in the crack extension plane of CT specimen at the load of $11.06 \mathrm{kN}$. 

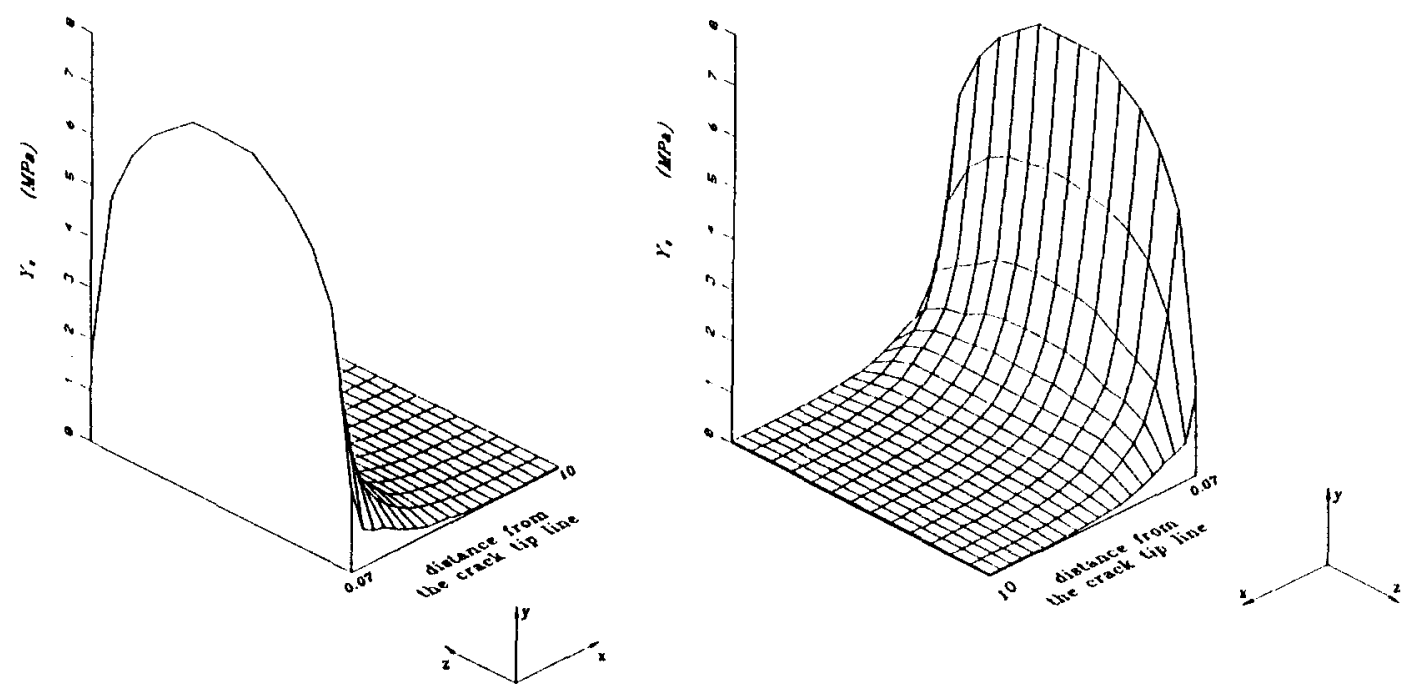

Fig. 9. Distribution of $Y_{R}$ in the crack extension plane of CT specimen at the predicted crack initiation load of $13.96 \mathrm{kN}$ with $Y_{R}$ criterion.

the fatigue precracking is conducted in an Amsler Vibrophore 10T high frequency fatigue testing machine. The chosen precracking fatigue load is small enough that no significant residual plastic deformation exists near the crack tip.

After the precracking, the CT specimens are loaded to different magnitudes in displacement control mode in an universal 10T MTS testing machine so that different crack propagations are created after the crack initiation. Then the crack profiles are marked by the heat-tint method and the specimens are reloaded to fracture. The load-crack propagation profile relation is thus obtained and load $P$-crack propagation length $\Delta a$ is obtained as shown in Fig. 3, where the crack propagation length $\Delta a$ is defined as the crack propagation area divided by the specimen thickness. From the $P-\Delta a$ data, the crack initiation load is determined to be $14.2 \mathrm{kN}$. Careful observation of the crack profiles after initiation reveals that the crack first initiates in the middle of the crack tip line. After the initiation, the crack propagates nonuniformly across the thickness. Its propagation is fastest in the middle, but lowest at the surface. Also it is found that the nonuniformity of crack propagation tends to increase during the crack propagation process. These phenomena suggest that a three-dimensional analysis of crack initiation and propagation is necessary in order to gain good understanding of the fracture process at the crack tip.

\section{Definition of three-dimensional failure criteria}

For thick specimens, the stress state across the thickness is nonuniformly distributed. At the specimen surface, a state of plane stress prevails, while in the interior part of the specimen, the stress state approaches the state of plane strain. For a thick specimen with a crack, the stress distribution is not uniform with high gradient in the crack tip region. Because the volume changes are negligible in the effective plastic strain space, yielding in tension can only occur when it is accompanied by a contraction in one or both perpendicular directions. Due to the compatibility in strain, the contraction is restrained by a large bulk of material far away from the crack tip at lower stress triaxiality, such that normal stresses in these directions are set 


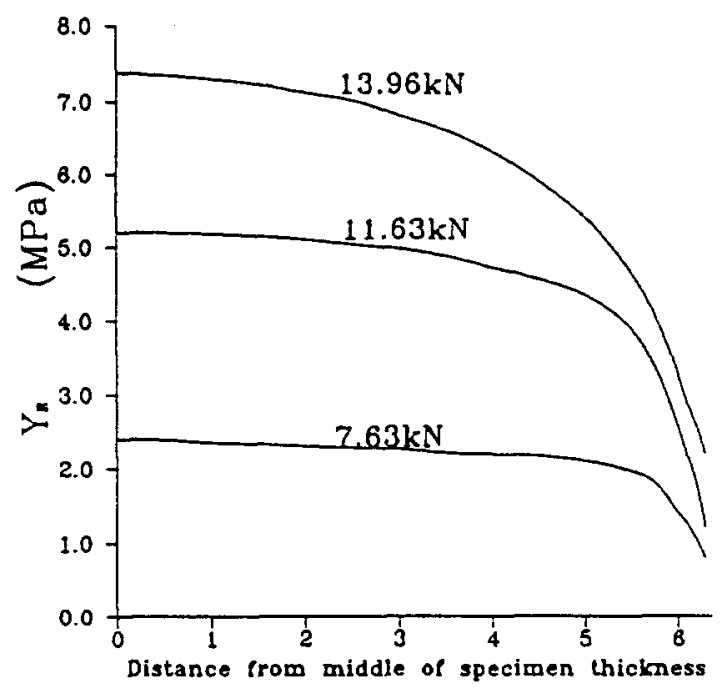

Fig. 10. Distribution of $Y_{R}$ at a constant distance of $0.07 \mathrm{~mm}$ from the crack tip line.

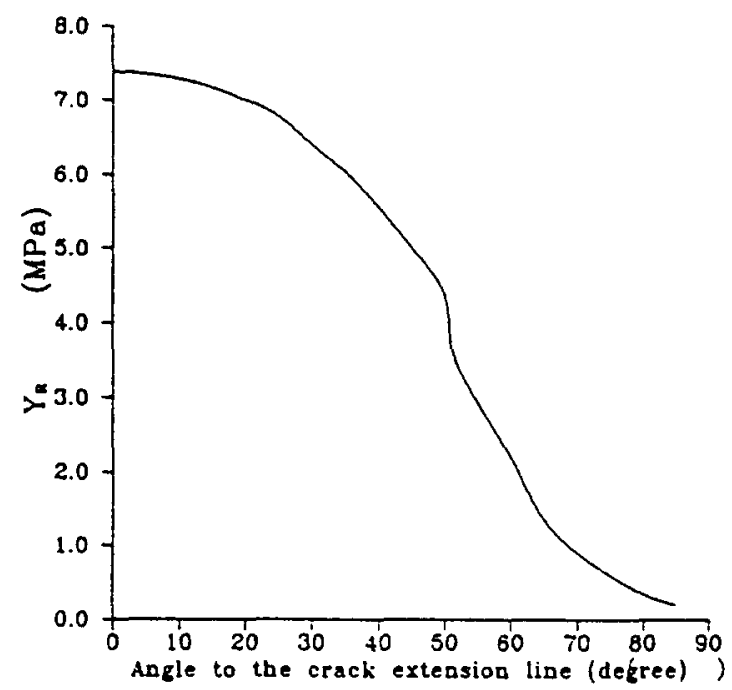

Fig. 11. The angular distribution of $Y_{R}$ in the crack tip of mid-plane.

up inducing high stress triaxiality in the near crack tip region. From the plasticity theory, a higher maximum tensile stress is necessary to cause yielding in a high triaxial stress state. As the deformation in the middle of the crack tip line is most constrained, the maximum stress triaxiality is therefore located there.

Since the damage evolution is highly dependent on the stress triaxiality, damage will accumulate much faster in the interior part of the specimen than on the surface, thus causing the first crack initiation in the interior part and the nonuniform crack propagation. These three-dimensional effects will be the main subject of discussion.

In a previous plane stress analysis of CCT specimens, local failure criteria were introduced [14]. Obviously the criteria proposed in the two-dimensional case cannot be directly applied to the three-dimensional case without suitable modification. 

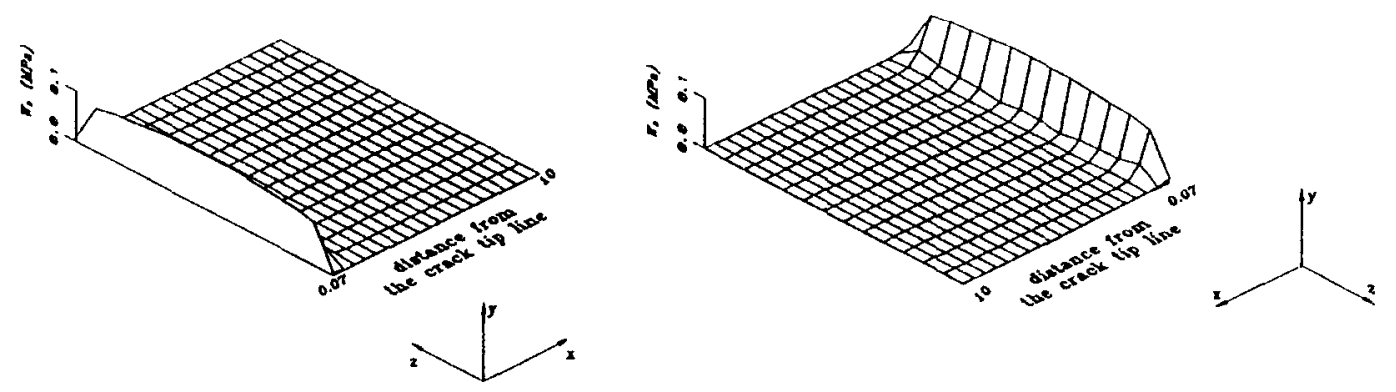

Fig. 12. Distribution of $W_{D}$ in the crack extension plane of CT specimen at the load of $11.06 \mathrm{kN}$.
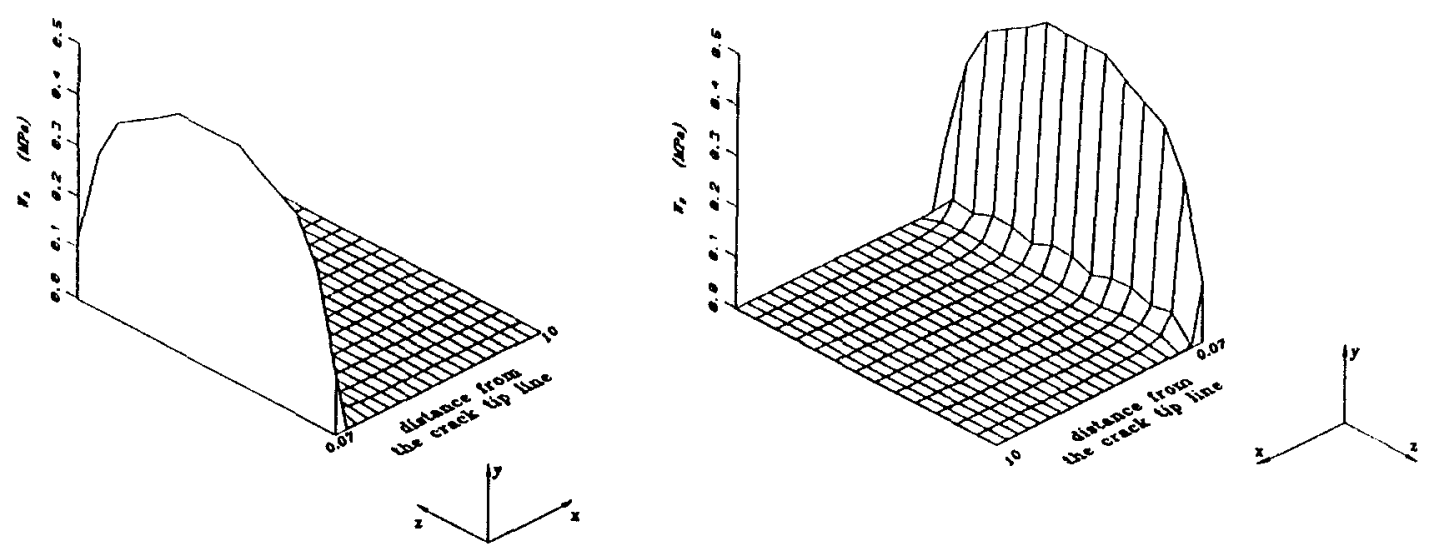

Fig. 13. Distribution of $W_{D}$ in the crack extension plane of CT specimen at the predicted crack initiation load of $13.8 \mathrm{kN}$.

The three-dimensional crack initiation criteria used in this study are defined in the following way. First, a plane perpendicular to the crack front line is assumed as shown in Fig. 4. As discussed in the two dimensional case, $Y_{R}$ is defined as the tangential component of damage strain energy release rate $Y$ at a radial distance from the crack tip (Fig. 5) where the crack will advance in the direction perpendicular to the crack front line. The $Y_{R}$ criterion assumes the crack initiates in the direction where the $Y_{R}$ attains its maximum value at the location where the maximum value $Y_{R}$ reaches its critical value at a characteristic distance from the crack tip in this plane. The critical value of $Y_{R}$ is $7.39 \mathrm{MPa}$ which is determined from the uniaxial tension test.

As to the second criterion, $\delta$, which is defined similarly to that in the two-dimensional case as shown in Fig. 6, is the angle between the radial line from the crack tip and the maximum damage plane. This parameter is used to determine the crack initiation direction. The crack is assumed to initiate in the direction where $\delta$ is the minimum. When the crack initiation direction is determined, the critical condition for the crack initiation along this direction is the damage dissipation energy $W_{D}$ defined as

$$
W_{D}=\int_{0}^{\zeta} \boldsymbol{Y}: \mathrm{d} D
$$

reaching its critical value at a characteristic length from the crack tip. The critical $W_{D}$-value determined from the uniaxial tension test is $0.46 \mathrm{MPa}$. 


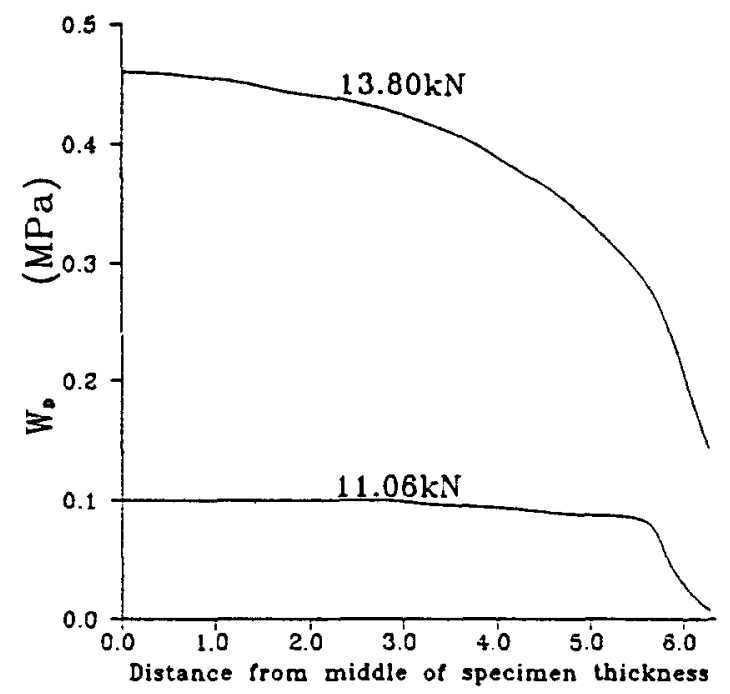

Fig. 14. Distribution of $W_{D}$ at a constant distance of $0.07 \mathrm{~mm}$ from the crack tip line.

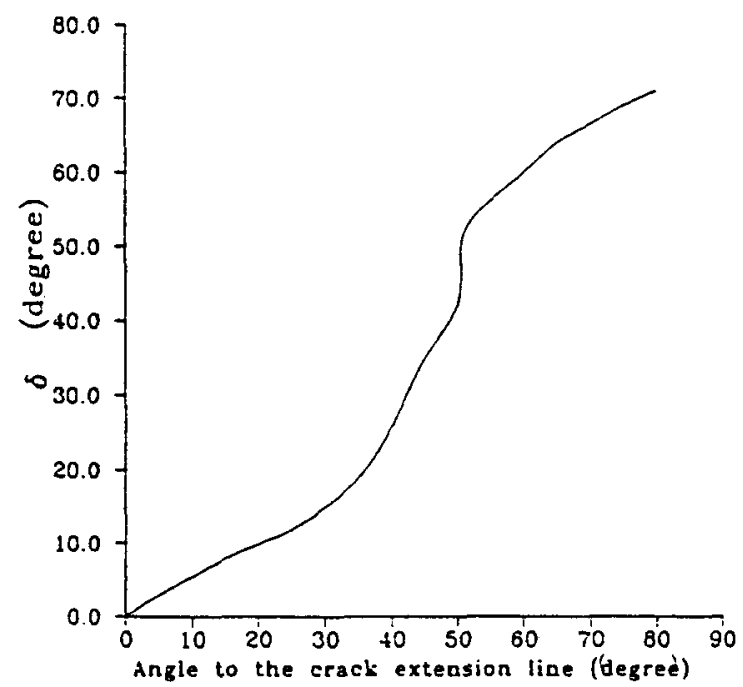

Fig. 15. The angular distribution of $\delta$ in the crack tip of mid-plane.

With the crack initiation criteria at one point in the crack front line defined above, the first step in three-dimensional analysis is to find the critical point along the crack front line. Then the failure criteria are employed to evaluate the crack initiation load in the plane perpendicular to the crack front line.

\section{Discussion of results}

From the numerical results, the crack initiation loads are evaluated using the $Y_{R}$ or the $W_{D}$ criterion. According to the $Y_{R}$ criterion, the predicted crack initiation site is located at the middle of the crack tip line along the direction of crack extension in the midplane. The initiation load predicted by the $Y_{R}$ criterion is $14.0 \mathrm{kN}$ where the characteristic length is $0.07 \mathrm{~mm}$. As to the $W_{D}$ and $\delta$ criterion, the characteristic length chosen is also $0.07 \mathrm{~mm}$. 

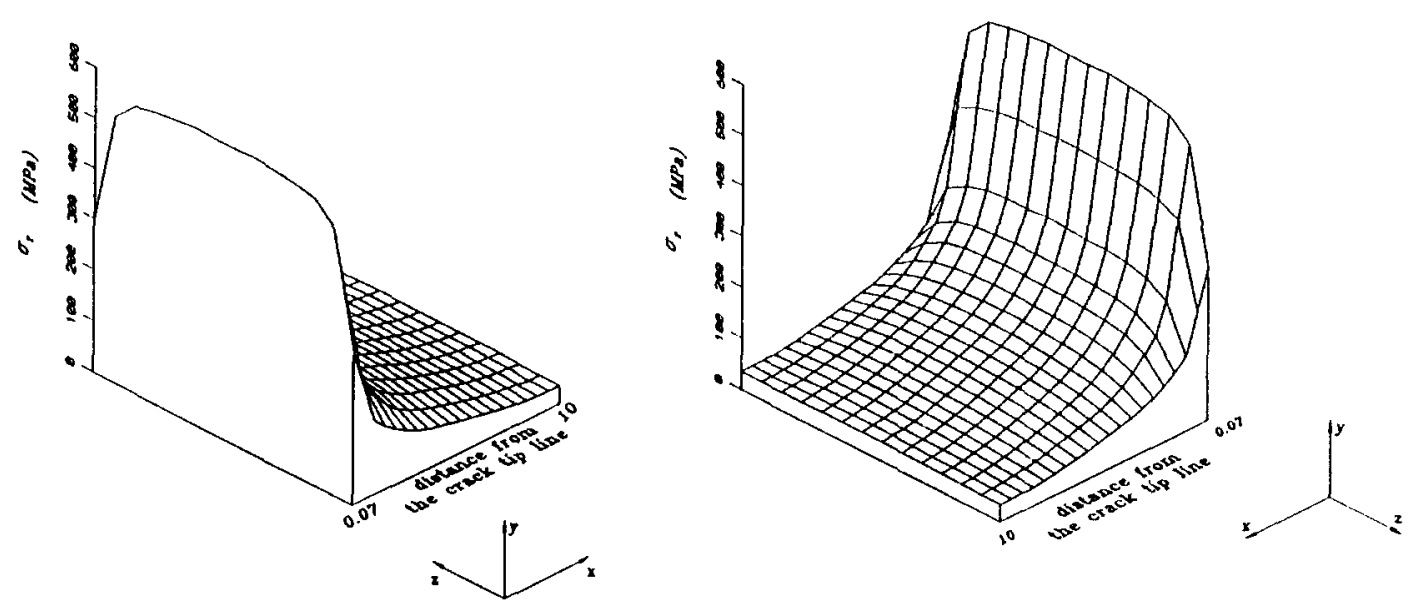

Fig. 16. Distribution of $\sigma_{y}$ over the crack extension plane of CT specimen at the load of $7.63 \mathrm{kN}$.

The predicted initiation site and direction are the same as those given by the $Y_{R}$ criterion, and the predicted initiation load is $13.8 \mathrm{kN}$. The initiation loads predicted by the $Y_{R}$ criterion and the $W_{D}$ criterion agree well with the measured value of $14.2 \mathrm{kN}$ described in Section 4 Experimental investigation.

Preliminary results from the three dimensional numerical analysis reveal that the stress, strain and other physical quantities are not uniformly distributed across the specimen thickness, especially near the crack tip line. This confirms that the three-dimensional analysis is necessary as the thickness of specimen chosen is not small enough that plane stress analysis is applicable and at the same time not large enough to attain the state of plane strain deformation in most interior parts of the specimen thickness. On the specimen surface, the state of plane stress prevails. The stress state approaches the plane strain state when the distance from the specimen surface to the mid-plane increases. This can also be observed from the stress triaxiality distribution diagram over the crack extension plane later.

From the numerical analysis, the $Y_{R}$ which first attains its critical value is the principal component of $Y$ perpendicular to the crack extension plane at the middle of the crack tip line. In Fig. 7, 8 and 9, the $Y_{R}$ distributions in the crack extension plane (the component of $Y$ perpendicular to the crack extension plane) at the applied loads of $7.63 \mathrm{kN}, 11.63 \mathrm{kN}$ and $13.96 \mathrm{kN}$ are shown respectively. When the load is small, the distribution of $Y_{R}$ across the thickness direction along the crack tip line is smoother. At a higher applied load, the variation rate of $Y_{R}$ across the thickness direction at the crack tip increases. The rate of increase in $Y_{R}$ to the applied load in the near crack tip region, especially in the middle plane, is at its maximum and the rate rapidly decreases as the distance from crack tip line increases. This indicates that $Y_{R}$ development is highly localized in the middle of the interior part of the crack tip region. The reason for this phenomenon is that $\sigma_{y}$ and stress triaxiality are high at the middle part of the crack tip line due to high deformation constraints across the specimen thickness, thus the rapid development of damage in this region.

The $Y_{R}$ distributions at the distance of $0.07 \mathrm{~mm}$ ahead of the crack tip line at three different loads are shown in Fig. 10. It can be observed from the figure that $Y_{R}$ distribution in the interior part is relatively uniform across the thickness at the lower load, but varies significantly close to the surface. As the load increases, the variation along the thickness direction of $Y_{R}$ increases considerably in both interior part and near the surface. 

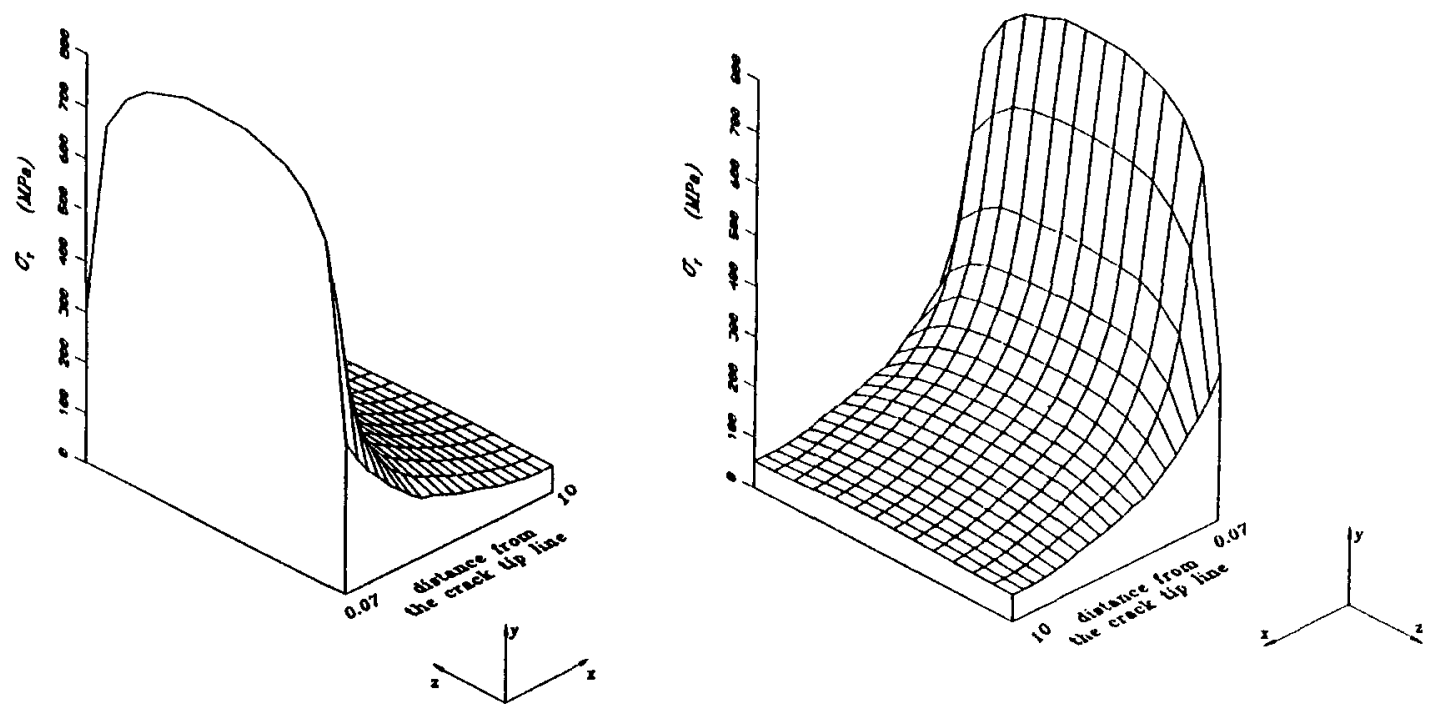

Fig. 17. Distribution of $\sigma_{y}$ over the crack extension plane of CT specimen at the load of $11.06 \mathrm{kN}$.

The $Y_{R}$ angular distribution at $r=0.07 \mathrm{~mm}$ in the mid-plane at the predicted crack initiation load of $13.96 \mathrm{kN}$ is shown in Fig. 11. The maximum value of $Y_{R}$ is located at $\theta=0^{\circ}$ to the crack extension line, where the crack is expected to propagate.

The $W_{D}$ distribution in the crack extension plane at the loads of $11.06 \mathrm{kN}$ and the predicted crack initiation load of $13.80 \mathrm{kN}$ are shown in Figs. 12 and 13, respectively. First, the non-zero $W_{D}$ is localized near the crack tip. This is because the plastic deformation is more localized in the crack tip region in this thick specimen than in the plane stress case due to the presence of high stress triaxiality. As shown in Fig. 14 which depicts the $W_{D}$ distribution along the thickness in the line $0.07 \mathrm{~mm}$ from the crack tip line, the variation of $W_{D}$ across the thickness direction in the interior part is modest for the lower load. As the load increases, the variation rate increases substantially at both the interior part and the surface.

The maximum value of $W_{D}$ is located in the middle of the crack tip line when it attains its critical value of $0.46 \mathrm{MPa}$ at the load of $13.80 \mathrm{kN}$. The $W_{D}$ criterion introduced above employs another variable $\delta$ to predict the crack initiation direction. The angular distribution of $\delta$ at $r=0.07 \mathrm{~mm}$ in the mid-plane is shown in Fig. 15. It is evident that the minimum value is obtained at $\theta=0^{\circ}$, indicating that the crack initiation direction is along the crack extension direction at the mid-plane.

In the above analysis, it is apparent that nonuniform crack initiation prevails. This results from the different stress and strain histories endured in the different elements across the crack front line. In the middle of the crack tip line, the stresses $\sigma_{y}$ and $\sigma_{z}$ reach their largest values, as does the stress triaxiality. Therefore the damage is most favorably developed at the location where the damage variables employed in the failure criteria such as $Y_{R}$ and $W_{D}$ attain their maximum values, and the crack is predicted to first initiate in the middle of the crack tip line. In what follows, the nonuniform distribution of $\sigma_{y}, \sigma_{z}$ and the stress triaxiality across the thickness are discussed.

In Fig. 16, the $\sigma_{y}$ distribution over the crack extension plane at the load of $7.63 \mathrm{kN}$ is shown. It remains constant for the most part of the crack tip line, but decreases significantly near the surface. As the distance from the crack tip line increases, $\sigma_{y}$ decreases rapidly and its 

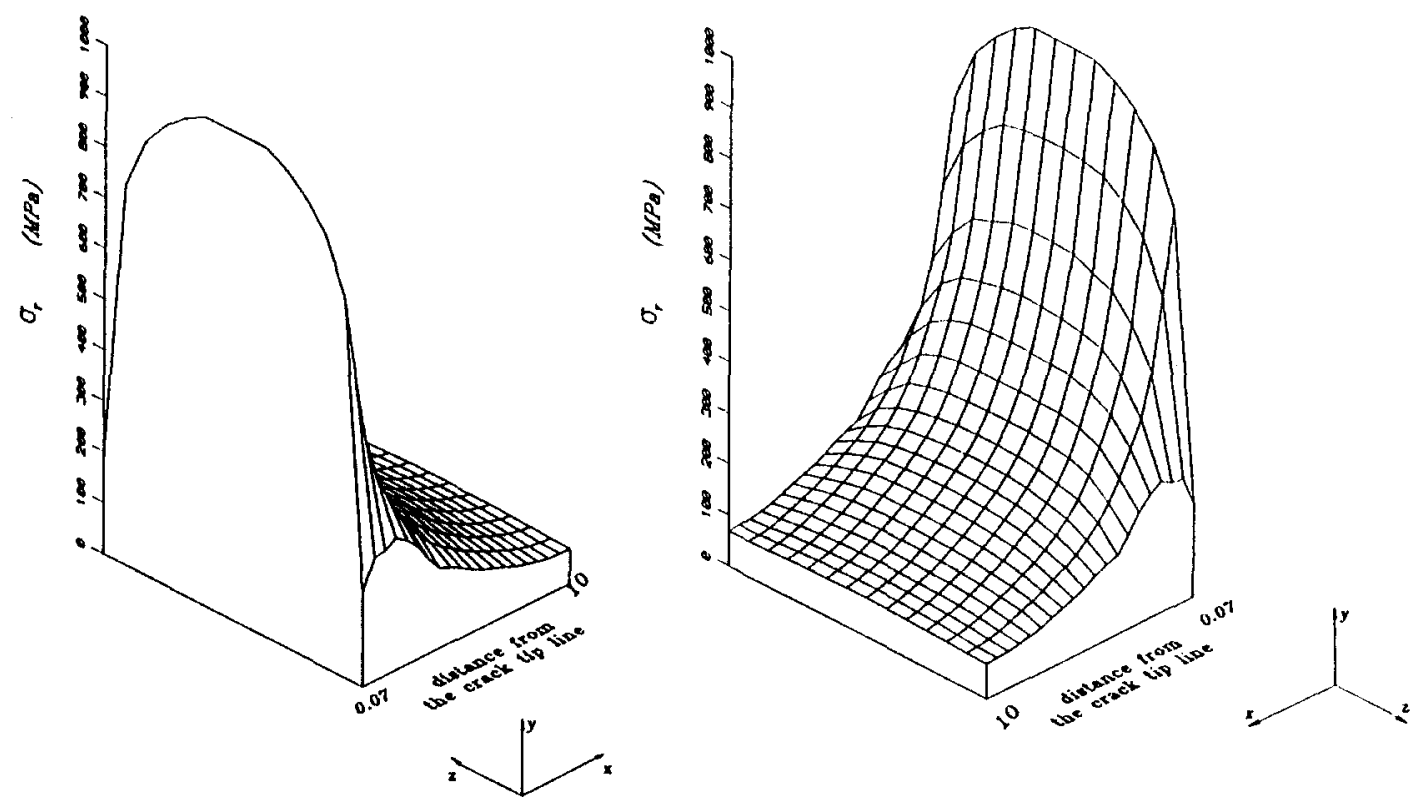

Fig. 18. Distribution of $\sigma_{y}$ over the crack extension plane of CT specimen at the predicted crack initiation load of $13.96 \mathrm{kN}$ with $Y_{R}$ criterion.
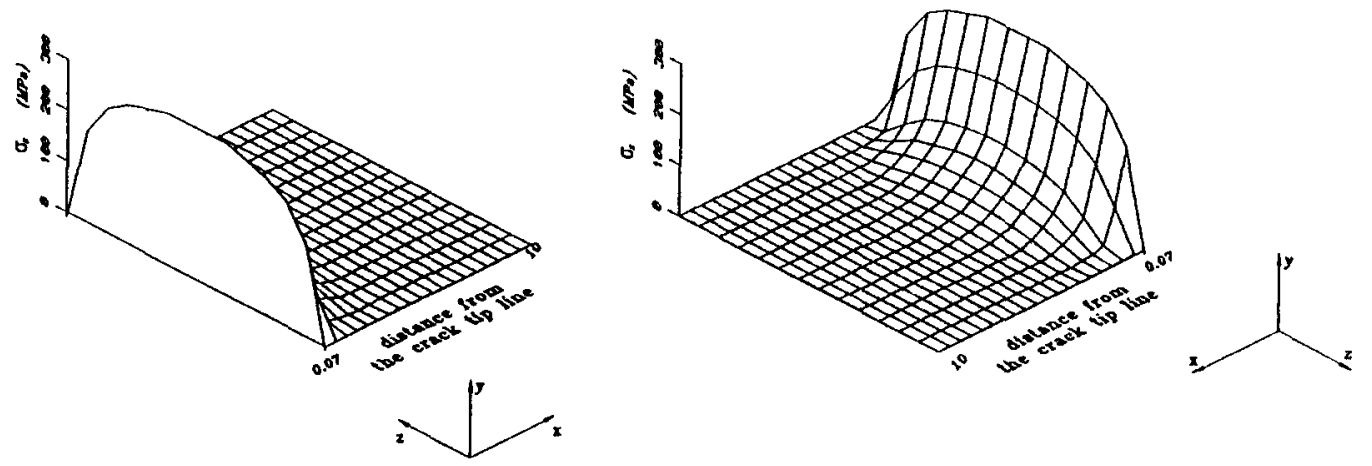

Fig. 19. Distribution of $\sigma_{z}$ over the crack extension plane of CT specimen at the load of $7.63 \mathrm{kN}$.
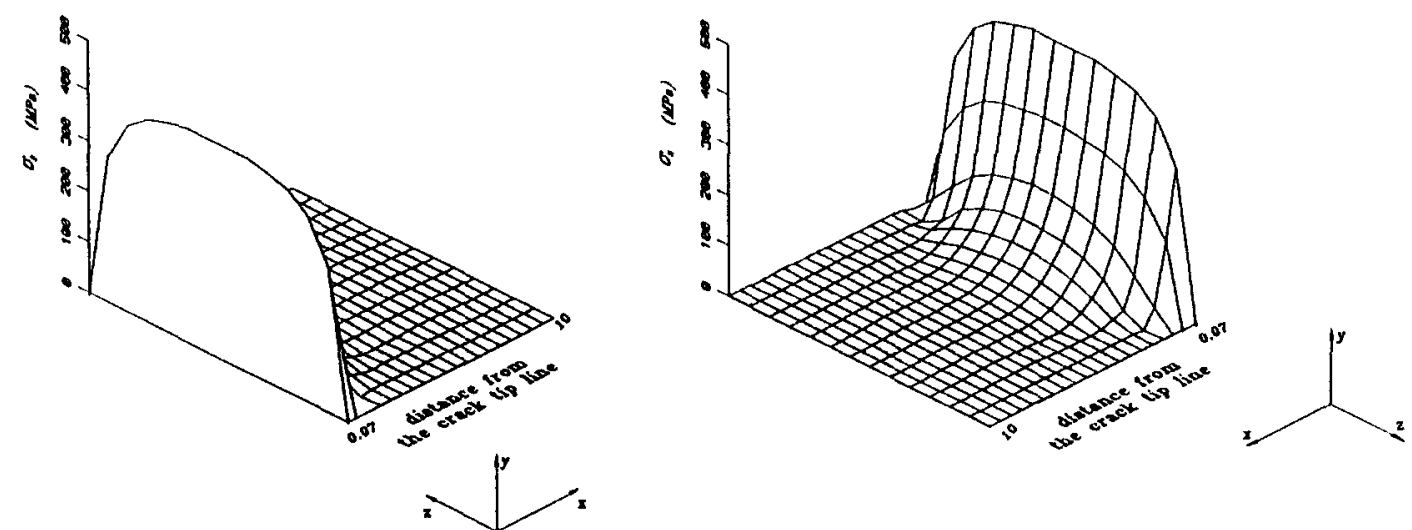

Fig. 20. Distribution of $\sigma_{z}$ over the crack extension plane of CT specimen at the load of $11.06 \mathrm{kN}$. 

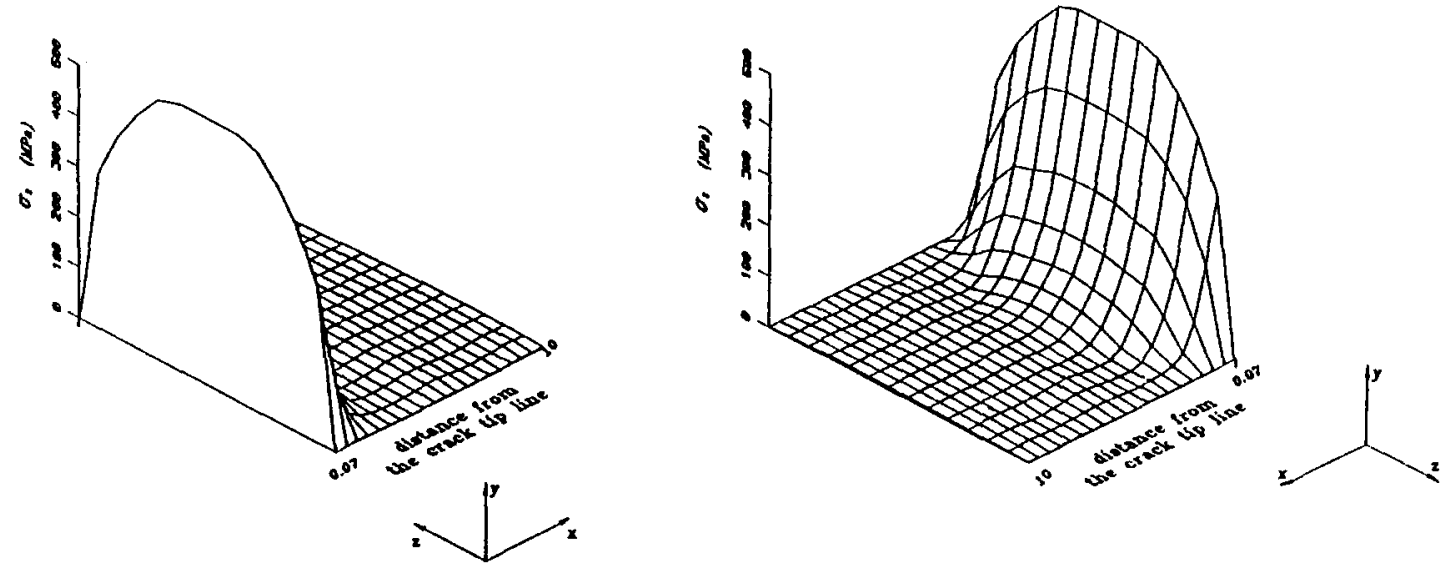

Fig. 21. Distribution of $\sigma_{z}$ over the crack extension plane of CT specimen at the predicted crack initiation load of $13.96 \mathrm{kN}$ with the $Y_{R}$ criterion.
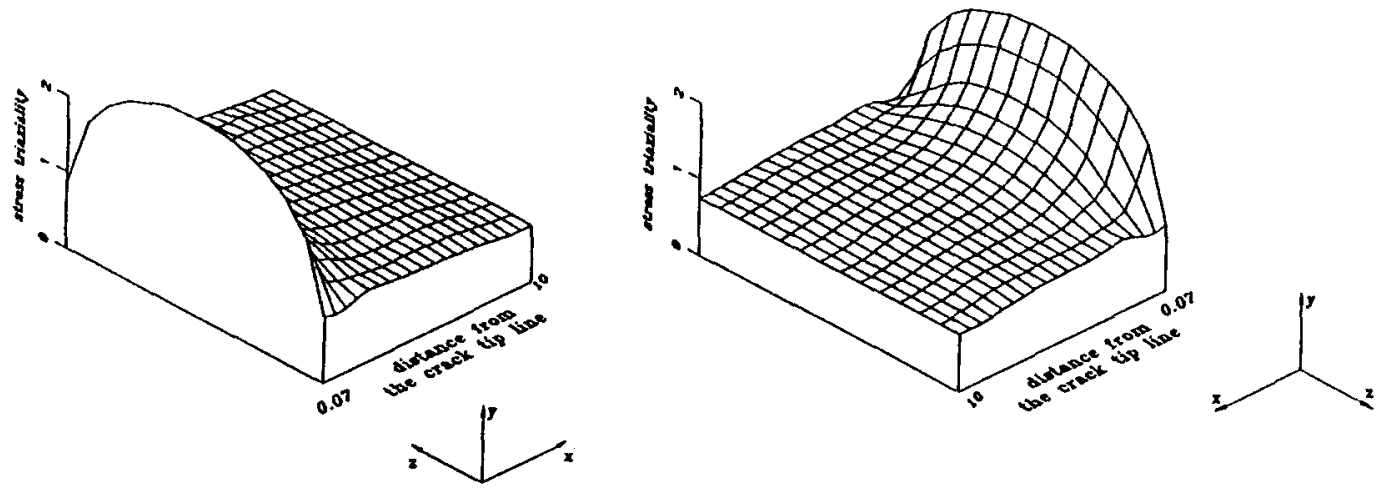

Fig. 22. Distribution of stress triaxiality over the crack extension plane of CT specimen at the load of $7.63 \mathrm{kN}$.

variation across the thickness diminishes to almost zero. The thickness effects are only severe at the crack tip, and decrease rapidly as the distance from the crack tip increases. In Fig. 17, when the applied load increases to $11.06 \mathrm{kN}$, the region where $\sigma_{y}$ remains almost constant in
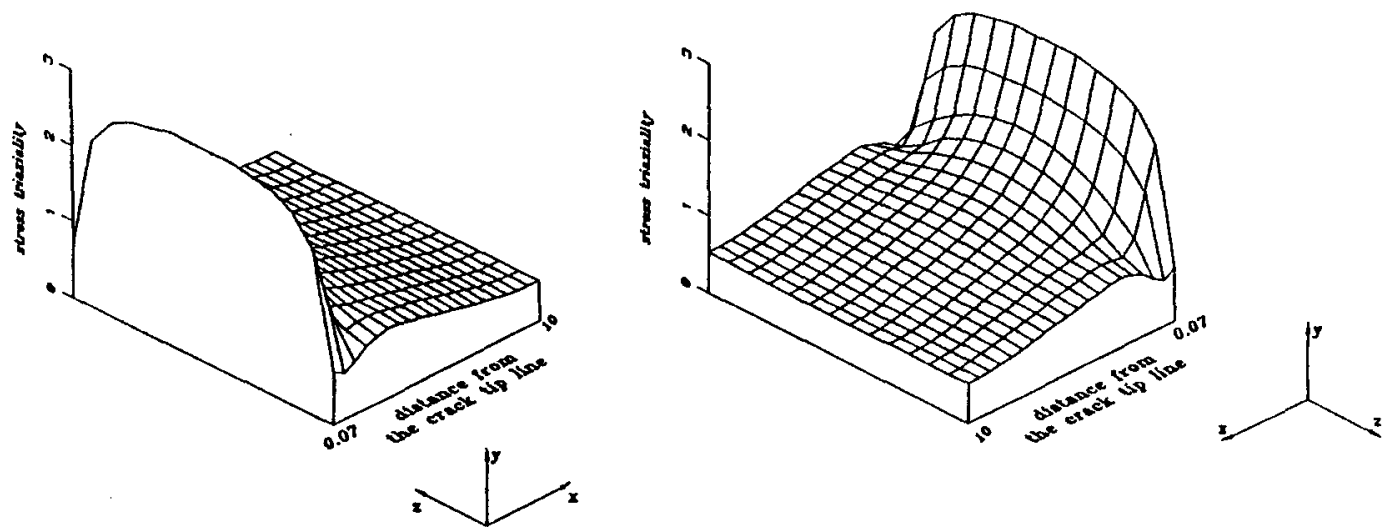

Fig. 23. Distribution of stress triaxiality over the crack extension plane of CT specimen at the load of $11.06 \mathrm{kN}$. 

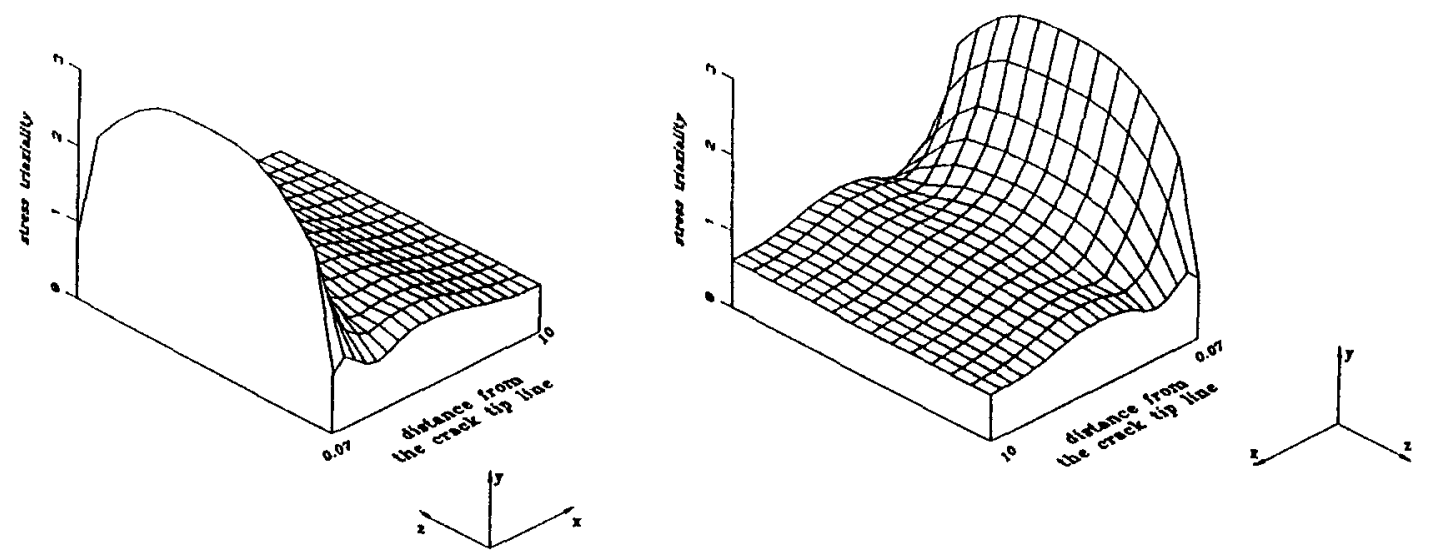

Fig. 24. Distribution of stress triaxiality over the crack extension plane of CT specimen at the predicted crack initiation load of $13.96 \mathrm{kN}$ with the $Y_{R}$ criterion.

the thickness direction decreases, and the variation of $\sigma_{y}$ across the thickness direction in the near crack tip region is also larger.

The $\sigma_{y}$ distribution over the crack extension plane at the predicted crack initiation load of $13.96 \mathrm{kN}$ with the $Y_{R}$ criterion is depicted in Fig. 18. It can be observed from the figure that the region of constant $\sigma_{y}$ in the crack tip line decreases further. Its variation along the crack tip line is larger than observed in both Figs. 16 and 17. This indicates that the three-dimensional effects increase with the increase in load.

The $\sigma_{z}$ distributions over the crack extension plane at the loads of $7.63 \mathrm{kN}, 11.63 \mathrm{kN}$ and $13.96 \mathrm{kN}$ are shown respectively in Figs. 19-21. As the load increases, the non-zero $\sigma_{z}$ zone changes modestly and is localized close to the crack tip line. Near the surface, $\sigma_{z}$ decreases very rapidly to zero where the boundary condition dictates. Therefore the size of the non-zero area along the crack extension direction is small close to the surface, relative to the interior part. Although the size of the non-zero $\sigma_{z}$ zone changes comparatively little during the loading process, the value of $\sigma_{z}$ changes significantly with the applied load.

As described in the damage evolution equation, the stress triaxiality plays a very important role in damage development. From Figs. 22-24 at their respective applied loads of $7.63 \mathrm{kN}$, $11.63 \mathrm{kN}$ and $13.96 \mathrm{kN}$, the maximum value of stress triaxiality is developed along the middle crack tip line. Parallel to the crack tip line, the stress triaxiality is at its maximum in the middle and decreases from the middle plane to the surface, and finally attains its minimum value at the surface. Closer to the crack tip line, the larger is the variation of stress triaxiality along the line parallel to the crack tip line. When the distance from the crack tip line reaches a certain value, the variation across the thickness diminishes to almost zero, which corresponds to the area where $\sigma_{z}$ is almost zero across the thickness.

\section{Conclusions}

In this paper, the three-dimensional effects of the thick CT specimen on the fracture analysis are examined. This is achieved by developing an endochronic plastic theory coupled with anisotropic damage and then the damage evolution equation from the orthogonality rule with the introduction of a newly defined intrinsic time scale for damage evolution. Then a 
damage model is employed to analyze the fracture of a thick CT specimen by means of the finite element method.

From the numerical results, the three-dimensional effects are pronounced. For the crack initiation analysis, $Y_{R}$ and $W_{D}$ distribute nonuniformly when the crack initiation criteria are satisfied in the middle of the crack tip line. This indicates that the crack will first initiate in the middle of the crack tip line, one of the significant three-dimensional effects in this analysis. The above phenomenon of the nonuniform crack initiation along the crack tip line results from the difference in the deformation constraint in the thickness direction, causing the nonuniform stress state and deformation state as well as plastic strain, stress triaxiality and other physical quantities.

During the loading process, the three-dimensional effects increase with the applied load. When the load is small, the distributions of stress, strain, etc. are more uniform as compared with those at a higher load. Their nonuniformity increases during the loading process until the threshold condition of crack initiation is reached in the middle of the crack tip line.

Experiments have been conducted to test a proposed endochronic damage model and the results are found to agree well with the predicted crack initiation loads and the crack initiation sites.

\section{References}

1. J.R. Rice, Journal of Applied Mechanics, Transactions of ASME (1968) 379-386.

2. J.W. Hutchinson, Journal of the Mechanics and Physics of Solids 16 (1968) 13-31.

3. J.R. Rice and G.F. Rosengren, Journal of the Mechanics and Physics of Solids 16 (1968) 1-12.

4. J.A. Begley and J.D. Landes, in Fracture Toughness, Proceedings of the 1971 National Symposium on Fracture Mechanics, Part II, ASTM STP 514 (1972) 1-20.

5. J.D. Landes and J.A. Begley, in Fracture Analysis Proceedings of the 1973 National Symposium on Fracture Mechanics, Part II, ASTM 560 (1974) 170-185.

6. K.B. Broberg, Journal of the Mechanics and Physics of Solids 19 (1971) 407-418.

7. D. Siegele and W. Schmitt, Computers and Structures 17, 5/6 (1983) 697-703.

8. D. Siegele, Computers and Structures 32, $3 / 4$ (1989) 639-645.

9. N.P. O'Dowd and C.F. Shih, Journal of the Mechanics and Physics of Solids 39, 8 (1991) 898-1015.

10. Ibid, 40, 5 (1992) 939-963.

11. F.A. McClintock, Journal of Applied Mechanics, Transactions of ASME (1968) 363-371.

12. C.L. Chow and J. Wang, Engineering Fracture Mechanics 30 (1988) 547-563.

13. C.L. Chow, Engineering Fracture Mechanics 33 (1989) 309-317.

14. C.L. Chow and X.F. Chen, International Journal of Fracture 60 (1993) 49-63.

15. K.C. Valanis, Arch. Mech. 23 (1971) 517-533.

16. Ibid, 23 (1971) 535-551.

17. K.C. Valanis, International Journal of Nonlinear Mechanics 6 (1971) 337-360.

18. R.S. Rivlin and J.L. Erickson, Journal of Rational Mechanics and Analysis 4 (1955) 323-425.

19. J.R. Rice and D.M. Tracey, Journal of the Mechanics and Physics of Solids 17 (1969) 201-217.

20. H. Ziegler, An Introduction to Thermomechanics, North-Holland, Series in Applied Mathematics and Mechanics (1977) Chapter 14.

21. C.L. Chow and X.F. Chen, International Journal of Fracture 55 (1992) 115-130. 